\title{
Die Höhenlage des Eises des Gletscherlobus während der Formung des Stauchwalles von Arnheim (Niederlande).
}

\author{
Frank Albert Peter de Zanger*)
}

Veluwe ice pushed ridge, erratics, granite, granodiorite, diorite, feldsparporphyre, gabbro, tuff, origin (Scandinavia) glacierlobe, Drenthe-stage, Saalian-age, glacial valley

Netherlands (Arnheim)

K u r z f a s s ung: Im Sommer 1977 wurde eine Untersuchung über das Auftreten von Kristallin-Geschieben (größer als $2 \mathrm{~cm}$ ) skandinavischen Ursprungs auf dem Stauchwall von Arnheim durchgeführt. Auf diesem Höhenzug, der in der ältesten Phase des Drenthe-Stadiums (Saalien) durch das Inlandeis aufgestaucht wurde, sind bis ca. $70 \mathrm{~m}$ N.A.P. nordische Findlinge vorhanden.

Es stellte sich heraus, daß auf den hohen Teilen des Stauchwalles keine nordischen erratischen Geschiebe vorkamen; man kann daraus schließen, daß nicht der ganze Stauchwall vom Eis eines Gletscherlobs überfahren worden ist, wie verschiedene Untersucher meinen. Der Stauchwall von Arnheim ist nur teilweise mit Eis von Gletscherzungen bedeckt gewesen.

In den konsequenten Tälern sind nordische Geschiebe gefunden worden; es sieht danach aus, daß viele dieser Täler in der Anlage glazial und darum von saalezeitlichem Alter sind.

Die Untersuchung auf dem Arnheimer Stauchwall macht eine Erklärung für die Erscheinung wahrscheinlich, daß auf bestimmten Stauchwällen (sowie der östliche Veluwe-Stauchwall) unterhalb eines bestimmten Niveaus $\mathrm{meh} \mathrm{r}$ nordische Gesteine vorkommen als oberhalb. Dieser Erklärung nach ist die Dichte der Bestreuung mit nördlichen Erratica an eine gewisse Stauungsphase gebunden.

\section{[The Altitude of the Ice of the Glacierlobe during the Formation of the Ice-pushed Ridge of Arnhem (the Netherlands)]}

Abstract: In the summer of 1977 research has been done concerning the presence of crystalline stones (bigger than $2 \mathrm{~cm}$ ) of Scandinavian origin on the ice-pushed ridge of Arnhem. On this chain of hills, that has been pushed by inland ice in the oldest phase of the Drenthe-Stage, northern erratics have been found up to a level of $70 \mathrm{~m}+$ N.A.P. It turned out that there were no northern erratics on the high parts of the ice-pushed ridge. The conclusion one can draw is, that not the whole ridge has been overridden by ice of a glacierlobe, as is the opinion of several researchers.

The ice-pushed ridge of Arnhem has only partly been covered by ice of glacierlobes. In the consequent valleys northern stones have been found. It appears that many of these valleys are originally glacial, and so of Saalian-age.

The research at the ice-pushed ridge of Arnhem provides a reasonable explanation for the phenomenon, that on certain ice-pushed ridges (as the eastern Veluwe-ice-pushed ridge) under a certain level more northern stones are found than above that level.

According to this explanation the closeness of the cover with northern erratics is related to a certain phase of ice-pushing.

\section{Einleitung}

Seit langem ist von Interesse, ob bestimmte Stauchwälle vom Landeis überfahren wurden oder nicht. Dies gilt in den Niederlanden insbesondere für die Gelände-Erhebungen im $\mathrm{N}$ des Landes. Von verschiedenen Forschern (z. B. Brouwer 1950) wird angenommen, daß sie nach der Formung überfahren wurden. TeR WeE (1962) dagegen ist der Meinung, wegen Anwesenheit glazialer Becken an Ort und Stelle, daß sie während des Rückzuges des Landeises entstanden.

*) Anschrift des Verfassers: Drs. F. A. P. de Zanger, p. a. Boomgaard 2, 2152 BP NieuwVennep, Niederlande. 


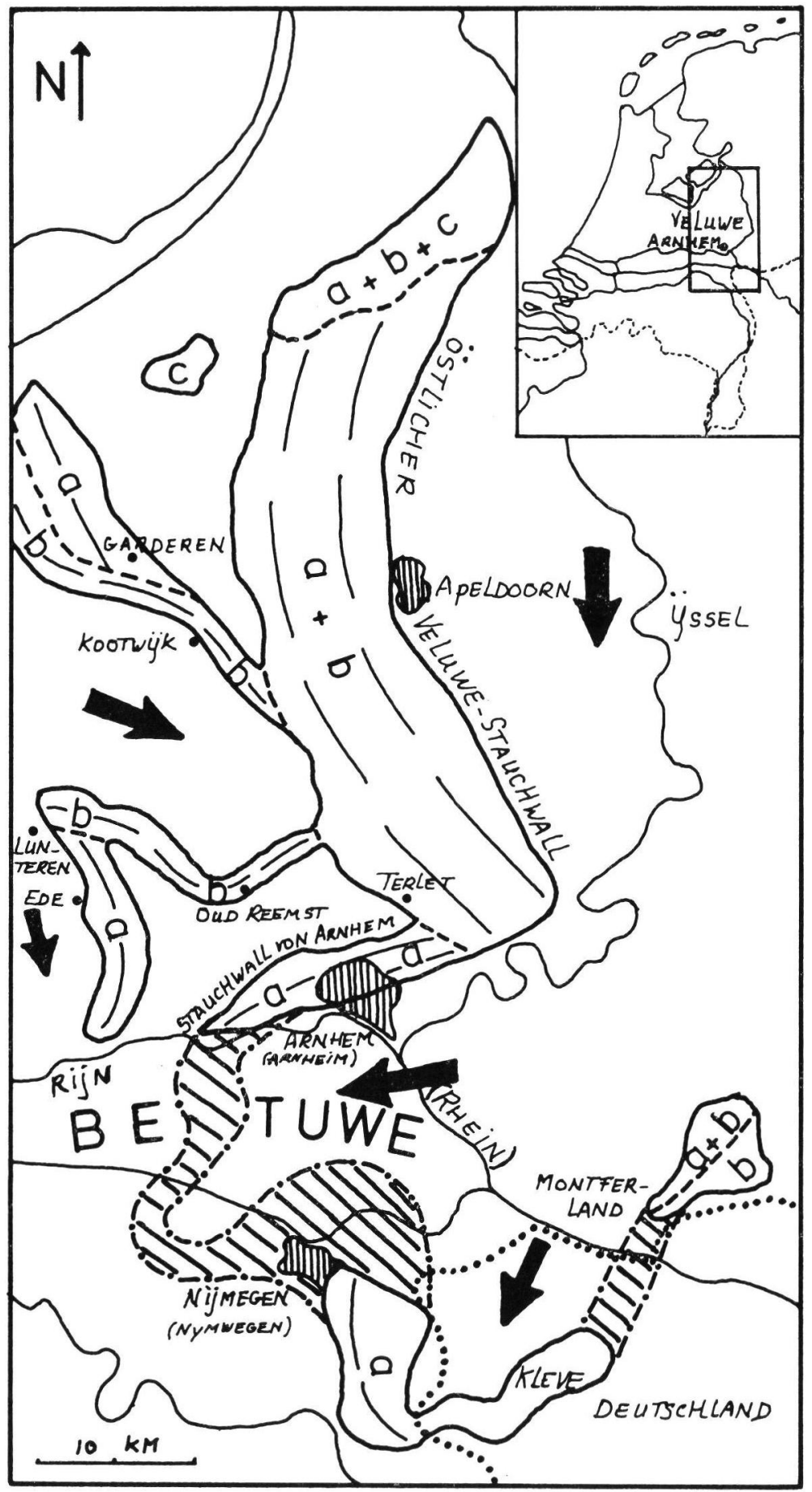

Abb. 1: Stauchwälle entstanden in Phase $a, b$ und $c$ in den Mittel-Niederlanden (nach MaARLEVELD, 1953, 1974). 
Dasselbe Problem gilt für die Stauchwälle der Rehburger Phase. Auch hierüber gehen die Meinungen stark auseinander (s. Duphorn 1974). Besonders die Anwesenheit von Geschiebelehm auf diesen Stauchwällen könnte ein Hinweis sein, daß diese so markanten Hügel ganz oder teilweise von Landeis bedeckt gewesen sind. Dies bedeutet aber nicht, daß sie von einer geschlossenen Eismasse überfahren sind, oder mit anderen Worten: es steht noch nicht fest, ob zusammenhängendes Inlandeis oder Eis eines Gletscherlobus oder Eis einer Gletscherzunge hierfür verantwortlich war.

Im Zusammenhang mit diesen Problemen wurde im Sommer 1977 eine Untersuchung über Vorkommen von Kristallin-Gestein (größer als $2 \mathrm{~cm}$ ) skandinavischen Ursprungs auf dem Stauchwall von Arnheim durchgeführt.

Dieser Stauchwall, der im Drenthe-Stadium des Saalien entstanden ist, wurde für diese Untersuchung ausgewählt wegen der einzigartigen Lage und außerdem, weil hier in den gestauchten Paketen kein nördliches Material vorkommt. Weiter sollte diese Untersuchung auf diesem Stauchwall einen Beitrag zu einem vollständigeren Bild der Entstehungsgeschichte der Veluwe-Stauchwälle liefern.

\section{Das Alter des Stauchwalles von Arnheim}

Aus einer Studien von MaArleveld (1953a) geht hervor, daß der Stauchwall von Arnheim in der ersten Stauchungsphase (Phase a) während des Drenthe-Stadiums gebildet wurde. Eine von $\mathrm{N}$ herkommende Eiszunge, die das damalige Rheintal benutzte (heutzutage das Isseltal, s. Abb. 1), schob teils in das heutige Gebiet der Betuwe hinein und bildete hierbei - hauptsächlich durch lateralen Druck gegen die Talwände - einen Stauchwallbogen. Dadurch entstanden die Stauchwälle von Arnheim, Nymwegen, Kleve und das Montferland (s. Abb. 1). Im Untergrunde sind diese Hügel noch miteinander verbunden (VERBRAECK 1975).

Nach Jelgersma \& Breeuwer (1975) dürfte der Stauchwall von Arnheim in einer späteren - von ihnen „zweite“ genannt - Stauchungsphase im Saalien gebildet worden sein. $\mathrm{Zu}$ dieser Schlußfolgerung kam man durch die Unterlagen über die Lagerung glazialer Becken im Untergrunde.

Untersuchungen von MAARLEveld (1953a), die auf vielen Messungen von Streichen und Fallen der zu Tage liegenden und aufgestauten Schichten beruhen, deuten darauf hin, daß der Stauchwall von Arnheim älter sein muß als der Stauchwall der östlichen Veluwe. Die Streichrichtung des östlichen Veluwe-Stauchwalles schließt nämlich nicht an den von Arnheim an; sie stehen sogar beinah senkrecht (MAARLEVELD 1953a) aufeinander, woraus auf einen Altersunterschied geschlossen werden kann (s. Abb. 1).

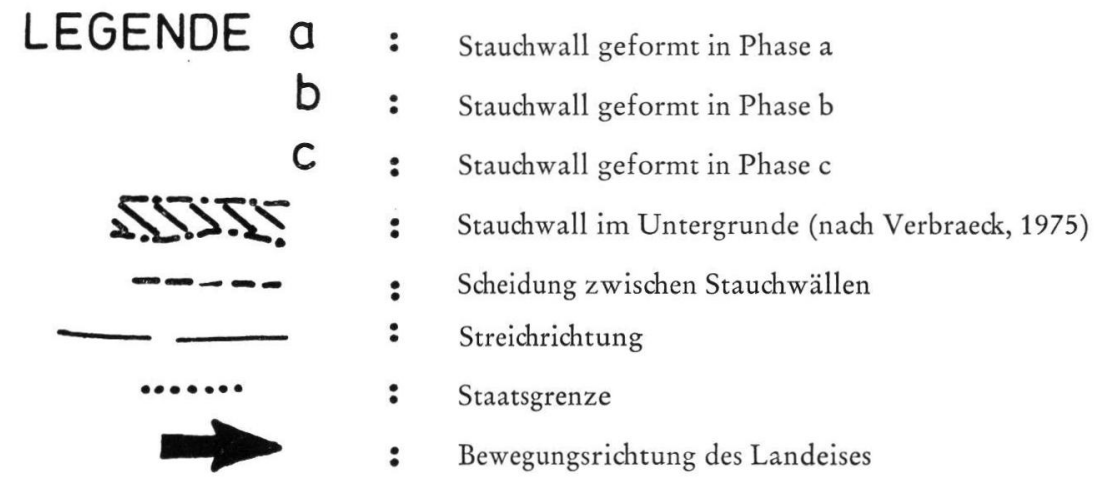


In einer zweiten Phase, in der das Inlandeis vorwärts drang und eine stauende Wirkung besaß (Phase b, MaArLeveld 1953a), wurde der Stauchwall von Arnheim nicht mehr berührt. Wohl wurde unter anderem der östliche Veluwe-Stauchwall zum zweiten Mal heraufgepreßt.

\section{Untersuchungs-Methode}

Der Stauchwall von Arnheim wurde systematisch auf das Vorhandensein nordischer Geschiebe untersucht. Ein Problem ist hierbei die Ungewißheit über die ursprüngliche Lage eines Gesteinsblockes und die ursprüngliche Dichte der Bestreuung, besonders in einem dicht bewohnten Gebiet.

Bei unseren Untersuchungen gingen wir wie folgt vor: Nur die nordischen Gesteine wurden als zuverlässig betrachtet, die in unverhärteten Wegen und Pfaden und auf Äckern gefunden wurden, und weiterhin diejenigen, die bei einem künstlichen Bach oder am Rande des Weges lagen und nicht zu künstlichen Wasserfällen, kleinen Brücken oder anderen deutlichen „Kunstwerken“ gehörten. Außerdem wurden die Funde der Mitglieder der „Nederlandse Geologische Vereniging“ dabei verwertet.

Es steht fest, daß früher mehr nordische Findlinge bzw. Geschiebe auf dem Stauchwall von Arnheim lagen als heutzutage. $Z u$ allerlei $Z$ wecken sind vor allem die größeren Exemplare weggeholt worden (MaArleveld 1950; VAN Der Lijn 1949). Dieses Problem konnte dadurch beseitigt werden, daß alle Steine größer als ca. $2 \mathrm{~cm}$ mit in die Untersuchung einbezogen wurden. Sind auch die großen Steine im Laufe der Zeit weggeholt worden, dann werden zweifellos kleinere liegen geblieben sein, die uns Information verschaffen können.

Auf dem Feld und bei der Determination im Laboratorium für Physikalische Geographie und Bodenuntersuchung an der Universität von Amsterdam wurde mit der Lupe gearbeitet. Für die Determination verschiedener Steine war eine Prüfung mittels Schleifplättchen nötig.

\section{Resultate}

Von den auf dem Stauchwall gefundenen erratischen Geschieben besteht mehr als die Hälfte aus Graniten. Weiterhin fand man Grano-Diorite, Diorite, Feldspat-Porphyre, Gabbro und Tuff.

Eine Anzahl großer Steine, die auf einem Plätzchen in dem Ort Oosterbeek aufgestellt sind, sind für die Untersuchung sehr wichtig. Diese Findlinge, von denen der größte - in der Längsrichtung in zwei Hälften gebrochen - 2,7 m lang und 1,2 $\mathrm{m}$ breit ist, wurden in 1896 beim Bau einer Villa (VAN CAPELle 1898) ausgegraben. Sie haben sehr flache, abgeschliffene Seiten, die auf einen Transport an der Basis des Eises hindeuten. Der Eislobus, der diese großen Steine herbeigeschafft hat, muß jedenfalls bis auf das Niveau der Fundstelle (ca. $50 \mathrm{~m}+$ N.A.P.) vorgerückt sein. In der Umgebung der Oosterbeekschen Blöcke wurden in 1977 noch verschiedene andere nordische Findlinge gefunden, worunter ein Granitblock in der Abmessung von $155 \times 90 \times 85 \mathrm{~cm}$ ist.

Außer den gerade erwähnten Granitblöcken in Oosterbeek sind noch eine Anzahl großer Steine in ansehnlicher Höhe gefunden worden:

\begin{tabular}{lrll} 
Granit & Länge & Höhe & Fundstelle \\
\hline$”$ & $100 \mathrm{~cm}$ & ca. $45 \mathrm{~m}+$ N.A.P. & Doorwerth \\
$"$ & $65 \mathrm{~cm}$ & ca. $45 \mathrm{~m}+$ N.A.P. & Rozendaal \\
$"$ & $90 \mathrm{~cm}$ & ca. $65 \mathrm{~m}+$ N.A.P. & Beekhuizen \\
$\#$ & $70 \mathrm{~cm}$ & ca. $50 \mathrm{~m}+$ N.A.P. & Beekhuizen \\
$"$ & $100 \mathrm{~cm}$ & ca. $55 \mathrm{~m}+$ N.A.P. & Velp
\end{tabular}


Auf Grund oben stehender Angaben kann angenommen werden, daß die Grundmoräne bis mindestens $60 \mathrm{~m}+$ N.A.P. auf dem Stauchwall abgelagert wurde und daß das Inlandeis im ganzen gewiß bis auf diese Höhe den Stauchwall bedeckt hat.

Aus der Verbreitung der Findlinge geht hervor, daß es lokale Unterschiede gibt. So beträgt die Maximumhöhe, bis zu der nordische Steine gefunden sind, auf dem östlichen Teil des Stauchwalles ca. $70 \mathrm{~m}+$ N.A.P. und auf dem westlichen Teil ca. $55 \mathrm{~m}+$ N.A.P. (s. Abb. 2).
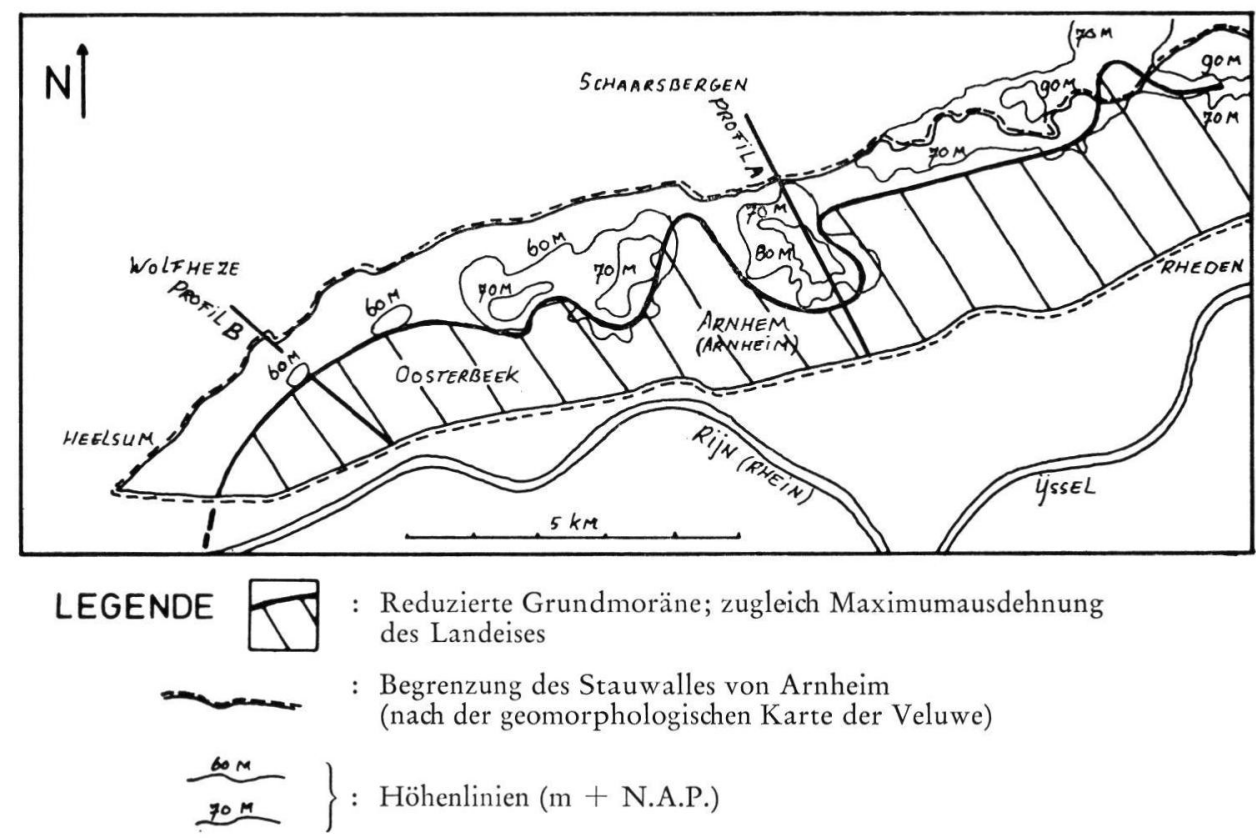

Abb. 2: Die Maximum-Ausdehnung des Landeises vgl. die Resultate der Untersuchung in 1977.

Die Oberfläche des Stauchwalles verläuft von maximal ungefähr $90 \mathrm{~m}+$ N.A.P. im E bis zu maximal ca. $60 \mathrm{~m}$ im W. Hieraus geht hervor, daß jedenfalls östlich des Ortes Oosterbeek das Eis nicht den ganzen Stauchwall überschritten hat, sondern vor dem heraufgepreßten Hügelrücken liegen geblieben ist (s. Abb. 3). Auf den hohen Kuppen des Arnheimer Stauchwalles sind keine nordischen Findlinge gefunden worden; diese hohen Teile werden also auch nicht mit Eis bedeckt gewesen sein. Nach dem Verbreitungsbild der Geschiebe zu urteilen ist das Eis bei einigen niedriger gelegenen Gebieten in Form von Eiszungen weiter durchgestoßen. Wie weit, ist wegen der späteren Bedeckung mit äolischen Sedimenten nicht festzustellen.

Ein gleichartiges Bild trifft man in der Nähe von Maarn an. Hier wurde Moränenmaterial in einem niedrig gelegenen Teil des Stauchwalles gefunden (FABER 1942). Der betreffende Stauchwall ist in Phase a geformt, doch ist er in Phase b gleichfalls mit dem Eis eines Gletscherlobus in Kontakt gewesen.

Westlich Oosterbeeks wird das Bild undeutlicher. Hier sind Geschiebe nicht nur auf der Südflanke des Stauchwalles gefunden worden, sondern einige auch auf der flachen Oberseite des Höhenzuges und sogar drei Exemplare auf der Nordflanke. In dem zuletzt 


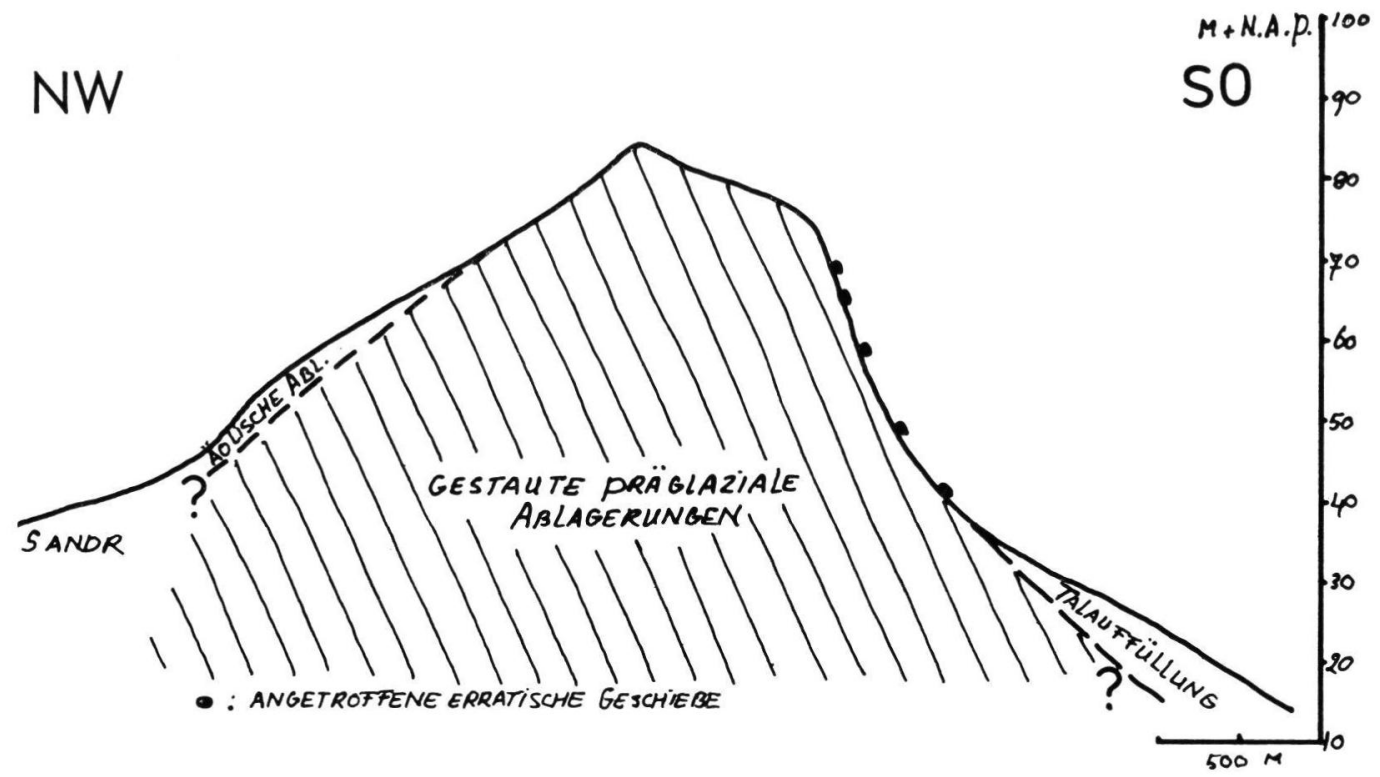

Abb. 3: Die Lage erratischer Geschiebe gegen den östlichen Teil des Stauchwalles von Arnheim (Profil A; siehe Abb. 2).

genannten Fall ist es nicht mehr möglich, die Gesteine, die von den Grundmoränen herstammen, von denen aus den fluvioglazialen Ablagerungen zu trennen (s. Abb. 4). Die flache Oberseite dieses Teiles des Stauchwalles (das sogenannte Stauchwall-Plateau) kann durch die abflachende Wirkung des obersten - horizontal sich bewegenden - Teiles des Eislobus, erklärt werden. KuENEN (1948) suggerierte eine derartige Lösung mit Bezug auf die flache Oberseite des östlichen Veluwe-Stauchwalles. Die Steinfunde lassen vermuten, daß die maximale Ausbreitung des Eises westlich von Oosterbeek nicht viel nördlicher gereicht hat als die Linie, die die Kuppen auf $60 \mathrm{~m}+$ N.A.P. verbindet.

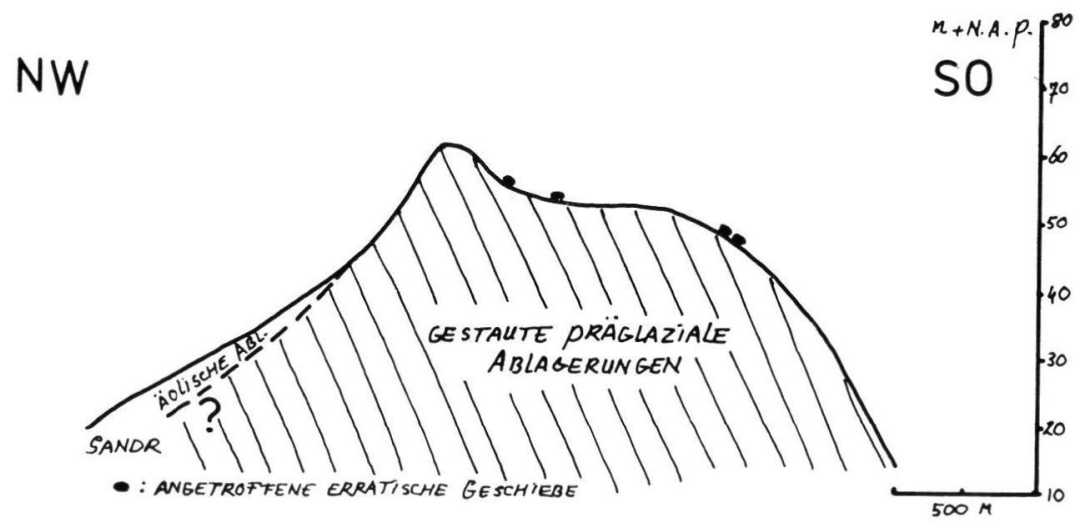

Abb. 4: Die Lage erratischer Geschiebe gegen den westlichen Teil des Stauchwalles von Arnheim (Profil B; siehe Abb. 2). 
Eine Tatsache ist weiterhin, daß die nordischen Geschiebe nicht auf den hohen Kuppen, sondern auf den tieferen Teilen des Stauchwalles gefunden wurden. Hier befinden sich auch die konsequenten Täler, und darum ist anzunehmen, daß viele dieser Täler im Ursprung glazigen sind. In den Vertiefungen des Stauchwalles, die im Weichselien vertieft und darauf teilweise wieder aufgefüllt sind (TEUNISSEN 1961), wurde dann auch bei Erdarbeiten ein Uberbleibsel der Grundmoräne in Form nordischer Geschiebe festgestellt. Diese Geschiebe müssen aus der sogenannten „Basis-Kiesschicht" herrühren (TeUnissen 1961), einem Niveau, in dem sich während des Überganges von der Erosions- nach der Auffüllungsphase der Täler grobes Material konzentriert hat und das größtenteils durch Abspülung entstanden ist.

Die Befunde stimmen nicht mit der Vorstellung überein, daß die ganze Veluwe, einschließlich des Stauchwalles von Arnheim, mit Eis bedeckt gewesen ist (Tesch 1927; TER WeE 1962). Diese Ansicht von der vollständigen Eisbedeckung geht aus der Erläuterung zu der Karte glaziale Erscheinungen während des Saalien hervor (Jelgersma \& BREEUWER 1975).

\section{Der Zusammenhang zwischen der Stauchungsphase und der Dichte der Gesteinsbestreuung}

Bei der detaillierten Untersuchung auf dem Stauchwall von Arnheim hat sich herausgestellt, daß große Steine $(90,70,55 \mathrm{~cm})$ bis auf die höchsten Stellen des Verbreitungsgebietes hinauf vorkommen, und daß außerdem die Ausbreitung der Gesteine über das Gebiet ziemlich regelmäßig ist. Es liegt kein Grund vor anzunehmen, daß die Art, wie Moränenmaterial sich auf dem Stauchwall von Arnheim abgelagert hat, anders ist als die auf anderen Stauchwällen, die in derselben Phase a (nach MAARLEveld 1953a) geformt sind.

Der Umstand, daß auf anderen Stauchwällen (u. a. der östliche Veluwe-Stauchwall) unterhalb eines gewissen Niveaus deutlich mehr nordisches Gestein anwesend ist als oberhalb, braucht dann auch nicht durch die Art und Weise von Transport des Moränenmaterials im Gletschereis erklärt zu werden, so wie Tesch (1927) und MaARleveld (1950) meinen. Es gibt eine andere Erklärung für dieses Phänomen. Wir verwenden hierbei neben den eigenen Untersuchungsergebnissen über den Arnheimer Stauchwall die Angaben von MaArleveld (1950) über die Dichte der Bestreuung mit nordischen Findlingen auf Teilen des Stauchwalles in Mittel-Niederland (die Stauchwälle von Oud Reemst, Ede und der östlichen Veluwe; s. Abb. 1).

MAARLEVeld macht hierbei Unterschied zwischen:

- Gebieten mit einer sehr verstreuten Lagerung (0-1 Exemplar pro $100 \mathrm{~m}^{2}$ )

- Gebieten mit einer kaum aneinanderstoßenden Lagerung (1-100 Ex. pro $\left.100 \mathrm{~m}^{2}\right)$.

- Gebieten mit einer aneinanderstoßenden geschlossenen Lagerung ( $>100$ Ex. pro $\left.100 \mathrm{~m}^{2}\right)$.

Die Gesteinsbedeckung auf dem Stauchwall von Arnheim fällt in Kategorie „sehr verstreute Lagerung" (0-1 Exemplar pro $\left.100 \mathrm{~m}^{2}\right)$.

Wenn der Zusammenhang schematisch wiedergegeben wird zwischen der Phase (oder den Phasen), in der (denen) ein Stauchwall gebildet ist und dem Maß der Bedeckung mit nordischen Gesteinen, dann entsteht die untenstehende Tabelle. Hierbei muß erwähnt werden, daß bei dem Stauchwall von Ede - nur nahe bei dem Kontakt mit dem jüngeren Stauchwall von Oud Reemst - eine abweichende Bestreuung vorhanden ist.

Es zeigt sich, daß die Stauchwälle, die im ganzen oder teilweise in der zweiten Stauchungsphase (Phase b) mit dem Eis in Berührung gewesen sind, mehr nordisches Material besitzen als die Stauchwälle (oder Teile davon), die nur in der ersten Phase (Phase a) durch Stauchung des Gletschereises beeinflußt sind. 


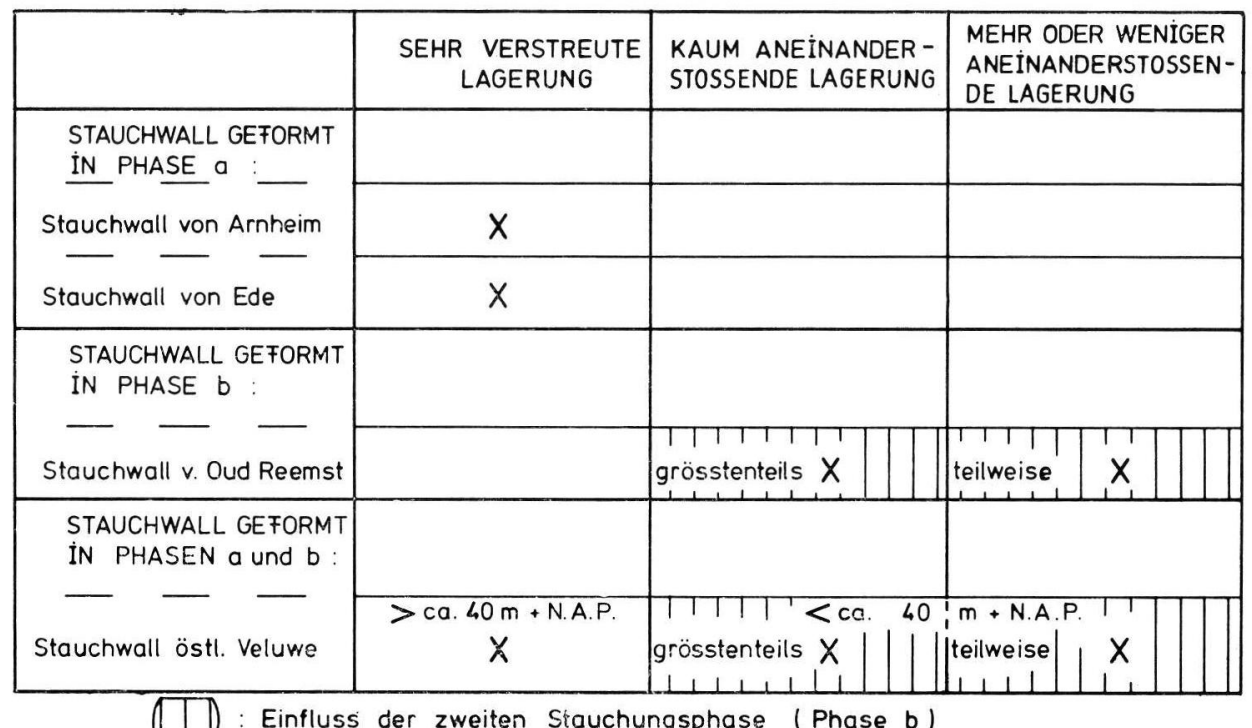

Tab. 1: Der Zusammenhang zwischen der Stauchungsphase und der Dichte der Bestreuung.

Die Geschiebe über ca. $40 \mathrm{~m}+$ N.A.P. auf dem östlichen Veluwe-Stauchwall können ein Restbestand der Grundmoräne sein, die in der ersten Stauchungsphase durch das Inlandeis abgesetzt wurde. Das Eis erreichte während dieser Phase seine maximale Ausdehnung und Dicke.

Nach MaArleveld (1950) werden Geschiebe bis über $80 \mathrm{~m}+$ N.A.P. im südlichen Teil des östlichen Veluwe-Stauchwalles angetroffen. Da dieser Höhenzug hier zweimal in die Stauchung einbezogen gewesen ist, kann aus der Höhenlage nicht die Konklusion gezogen werden, daß das Eis diese Höhe erreicht hat, weil das Moränenmaterial aus der ersten Stauchungsphase während der nächsten Phase hochgedrückt sein kann. Diese Bedenken gelten nicht für die Stauchwälle von Ede und Arnheim. Hier wurde Moränenmaterial bis zu einer Höhe von ca. $50 \mathrm{~m}$ (MaArleveld 1950) und ca. $70 \mathrm{~m}+$ N.A.P. sedimentiert.

Die Dichte der Gesteinsbestreuung auf den drei genannten Stauchwällen (östlicher Veluwe-Stauchwall: üb e r ca. $40 \mathrm{~m}+$ N.A.P.) ist vergleichbar; sie fällt in die Kategorie: „sehr verstreute Lagerung" (0-1 Ex. pro $\left.100 \mathrm{~m}^{2}\right)$.

Während der zweiten Phase der Stauchung (Phase b) erreichte das Gletschereis die Stauchwälle von Arnheim und von Ede also nicht mehr (MaArleveld 1953a). Es blieb in diesen Gebieten bei einer einmaligen Sedimentation der Grundmoräne. In Phase b entstand u.a. der Stauchwall von Oud Reemst (s. Abb. 1). Der Nordpunkt des heutigen Stauchwalles von Ede (bei Lunteren) kam hierbei zum zweiten Mal mit dem Eis in Berührung, und nordisches Material bedeckte dies Gebiet zum zweiten Mal.

Wie bereits erwähnt wurde, ist der östliche Veluwe-Stauchwall in der zweiten Phase aufs neue durch das Inlandeis aufgestaucht. Das Eis wird das Moränenmaterial hauptsächlich bis zu ca. $40 \mathrm{~m}+$ N.A.P. abgesetzt haben. Diese zweite Bedeckung von Grundmoräne muß, wenn dieser Gedankengang richtig ist, der Grund dafür sein, daß b is zu $40 \mathrm{~m}+$ N.A.P. die Dichte der Gesteinsbestreuung deutlich größer ist als in dem Gebiet oberhalb der 40-m-Höhenlinie. 
Aus obenstehenden Angaben geht hervor, daß bei der Untersuchung der Dichte der Bestreuung mit nordischen Findlingen auf Stauchwällen mit einigen Faktoren gerechnet werden muß, denen bis jetzt wenig Aufmerksamkeit geschenkt wurde:

1) Dem Faktor Zeit. Die ältesten Stauchwälle waren am längsten der Erosion ausgesetzt, wodurch auch das meiste nordische Material abgeführt sein wird.

2) Auf Stauchwällen, die zum zweiten Mal (oder mehrere Male) durch das Inlandeis aufgestaut sind, wird - durch diesen wiederholten Kontakt - auch meh r nordisches Gəstein abgesetzt sein.

\section{Konklusionen}

Aus der Verbreitung und den Eigenschaften der nordischen Findlinge auf dem - währen e i n e $r$ Phase geformten - Stauchwall von Arnheim kann konkludiert werden, daß - während der maximalen Ausbreitung des Eises (vom Gletscherlobus) der Stauchwall nicht überfahren, aber teilweise mit Eis bedeckt gewesen ist,

- lokal - in einigen niedrigen Teilen - das Eis von Gletscherzungen über den Stauchwall geflossen ist,

- die heutige Morphologie des Stauchwalles stark bestimmt ist sowohl durch den Druck des Eises von einem großen Gletscherlobus als auch durch den von kleineren Eiszungen,

- die konsequenten breiten Täler auf dem Stauchwall in der Anlage von Saalien-Alter sein werden (danach sind diese Täler im Weichselien teilweise in der Form verändert),

- nordische Findlinge bis zu ca. $70 \mathrm{~m}+$ N.A.P. gefunden sind.

Soweit bekannt ist, stimmt die Dichte der Bestreuung mit nordischen Findlingen auf den Stauchwällen der Veluwe, die gleichen Alters sind, überein. Stauchwälle, die in der ersten Stauchungsphase entstanden sind, sind momentan in deutlich geringerem Maße mit skandinavischen Gesteinen bedeckt als Stauchwälle, die in der zweiten Phase gebildet worden sind. Die Konklusion liegt nahe, daß Erosion in der dazwischen liegenden Periode diesen Unterschied hauptsächlich verursacht haben muß.

Mein Dank gilt besonders Herrn Professor Dr. G. C. MaAnleveld, unter dessen Leitung die Untersuchung stattfand, für seine Hinweise zur Verbesserung des Manuskriptes.

Weiterhin danke ich: Herrn Dr. J. Verhorstad für seine Hilfe bei der Determination der gefundenen nördlichen Erratica, dem „Nederlandse Geologische Verenigung“ für die Zurverfügungstellung von Unterlagen, und Frau A. Otтer-Erasmus, die die Übersetzung aus dem Holländischen ins Deutsche auf sich nahm.

\section{Schriftenverzeichnis}

Boulton, G. S. (1972): Modern Arctic glaciers as depositional models for former ice sheets. II. geol. Soc. London, 128: 361-393; London.

Brouwer, A. (1950): De glacigene landschapstypen in oostelijk Midden-Nederland. - Tijdschr. Kon. Ned. Aardr. Gen., 67: 20-32; Amsterdam.

Capelle, H. van (1898): Nieuwe waarnemingen op het Nederlandsche diluviaalgebied. - Meded. omtrent de Geologie van Ned., 23; Verh. Kon. Ak. van Wet. Amsterdam, 2 de sectie, deel VI, no 2.; Amsterdam.

CraAts, S., van DE (1966): Anderhalve eeuw dak van Oosterbeek; Van Jagers, Herders en Atleten; 1966.

FABER, F. J. (1942): Nederlandse landschappen. - 240 S., Gorinchem.

JELgERSMA, S. \& BREeuwer, J. B. (1975): Toelichting bij de kaart glaciale verschijnselen gedurende het Saalien, 1:600 000; Toclichting bij geologische overzichtskaarten van Nederland, Rijks Geologische Dienst, Haarlem.

Jong, J. D. DE (1967): The Quarternary of the Netherlands. - In: Rankama, K.: The quarternary, 2: 301-426; New York - London - Sydney (Interscience Publishers). 
Kuenen, Ph. H. (1948): De Kringloop van het water. - Den Haag (H. P. Leopolds uitgeversmaatschappij N. V.).

LiJn, P. van DER (1949): Het keienboek. Inleiding bij de studie onzer zwerfsteenen. - 3. Aufl., 327 S.; Zutphen (Thieme \& Co.).

MaArleveld, G. C. (1950): Iets over de verspreiding van noordelijke zwerfstenen op en nabij de stuwwallen in midden-Nederland. - In: Sporen der ijstijd: 71-79; Zutphen (Thieme \& Cie.).

- (1953a): Standen van het landijs in Nederland. - Boor en Spade, VI: 95-105; Wageningen.

- (1953b): De geologische geschiedenis van de zuidelijke Veluwe. — Boor en Spade, VI: 105112; Wageningen.

- (1974): Atlas van Nederland, blad III - 1. - s'Gravenhage (Staatsdrukkerij en Uitgeverijbedrijf).

Martin, K. (1889): Het eiland Urk benevens eenig algemeene beschouwingen over de geologie van Nederland. - Tijdschr. Kon. Ned. Aard. Gen. Amsterdam, 2de Ser., VI: 1-37; Leiden.

Tesch, P. (1927): De glaciale kneding. - Tijdschr. Kon. Ned. Aardr. Gen. Amsterdam, 2de serie, XLIV: $325-334$; Leiden.

Teunissen, D. (1961): Het middennederlandse Heuvelgebied. - Nat.wiss. Diss. Utrecht, 5. 6. 61. XIV + 153 S., Nymwegen (Janssen).

VEN, A. J. VAN DE (1933): De oude buitenverblijven rondom de stad. - Uit: Arnhem, zeven eeuwen stad; officieel gedenkboek; uitgegeven in opdracht van het Arnhemsch genootschap van oudheidkunde, met medewerking van het herdenkingscomité; Arnhem (Hijman, Stenfert Kroese en Van der Zanden).

Verbraeck, A. (1975): Ice-pushed ridges in the eastern part of the Netherlands river area. Geologie en Mijnbouw, 54: 82-84; s'Gravenhage.

Wee, M. W. Ter (1962): The Saalian Glaciation in the Netherlands. - Meded. Geol. Stichting, N. S., 15: 57-76; Haarlem.

Woldstedt, P. \& Duphorn, K. (1974): Norddeutschland und angrenzende Gebiete im Eiszeitalter. 3. Aufl. - 500 S.; Stuttgart (Koehler).

Zagwijn, W. H. (1973): Pollenanalytic studies of Holsteinian and Saalian Beds in the Northern Netherlands. - Med. Rijks Geol. Dienst, N. S., 24: 139-155; Haarlem.

- (1975): Indeling van het kwartair op grond van veranderingen in vegetatie en klimaat; Toelichting bij geologische overzichtskaarten van Nederland; 109-114; onder redactie van W. H. Zagwijn en C. J. Van Staalduinen, Rijks Geologische Dienst, Haarlem; 1975.

Manuskript eingegangen am 1. 6. 1979. 